\title{
Biodegradable implants for orbital wall fracture reconstruction
}

\author{
Hyeon Uk Jang, \\ So Young Kim \\ Department of Plastic and \\ Reconstructive Surgery, Inje University \\ Sanggye Paik Hospital, Inje University \\ School of Medicine, Seoul, Korea
}

\begin{abstract}
Background: Due to the different handling properties of unsintered hydroxyapatite particles/poly-L-lactic acid (UHA/PLLA) and polycaprolactone (PCL), we compared the surgical outcomes and the postoperative implantation accuracy between uHA/PLLA and PCL meshes in orbital fracture repair.

Methods: Patients undergoing orbital wall reconstruction with PCL and UHA/PLLA mesh, between 2017 and 2019, were investigated retrospectively. The anatomical accuracy of the implant in bony defect replacement and the functional outcomes such as diplopia, ocular motility, and enophthalmos were evaluated.

Results: No restriction of eye movement was reported in any patient ( $n=30$ for each group), 6 months postoperatively. In the PCL group, no patient showed diplopia or enophthalmos, while the UHA/PLLA group showed two patients with diplopia and one with enophthalmos. Excellent anatomical accuracy of implants was observed in 27 and 22 patients of the PCL and UHA/PLLA groups, respectively. However, this study showed that there were neither any significant differences in the surgical outcomes like diplopia and enophthalmos nor any complications with the two well-known implants.

Conclusion: PCL implants and UHA/PLLA implants are safe and have similar levels of complications and surgical outcomes in orbital wall reconstruction.
\end{abstract}

Keywords: Absorbable implants / Enophthalmos / Orbital fractures

\section{INTRODUCTION}

The sequelae of orbital wall fractures include diplopia and restriction of ocular movements caused by the entrapment of the extraocular muscle and soft tissue and enophthalmos caused by bony defect. Hence, the surgical treatment of this fracture aims to release the tissue entrapment or compression, reduce the prolapsed tissue/bone, and anatomically replace the bony defect

\section{Correspondence: So Young Kim}

Department of Plastic and Reconstructive Surgery, Inje University Sanggye Paik Hospital, Inje University School of Medicine, 1342 Dongil-ro, Nowon-gu, Seoul 01757, Korea

E-mail: pajamakim@naver.com

This work was supported by the 2018 Inje University research grant.

Received January 1, 2020 / Revised February 4, 2020 / Accepted February 20, 2020
[1]. Generally, alloplastic orbital implants are classified as biodegradable and non-biodegradable materials. Non-resorbable alloplastic materials, such as titanium, porous polyethylene (Medpor, Kalamazoo, MI, USA) and silicone elastomers, provide good tensile strength and maintenance because of their property of minimal or no resorption. However, these materials remain as permanent foreign bodies in vivo, and are susceptible to infection, bleeding, migration, and exposure over time [2].

With the recent popularity of biodegradable and biocompatible alloplastic materials, various biodegradable orbital implants that have the advantages of easy availability, biocompatibility, and low susceptibility to infections have been introduced in the clinical field. These include copolymers of poly-L-lactic acid (PLLA), poly-D-lactide (PDLLA), polyglycolic acid (PGA), 
polydioxanone (PDS), and polycaprolactone (PCL) of varying compositions. Except for PDS, which exhibited rapid degradation (in approximately 6 months) resulting in enophthalmos or hypophthalmos [3], various kinds of polylactide- and polyglycolide-derived implants have been popularly used as they show excellent mechanical properties with a relatively slow resorption period of 1-5 years based on their composition [4,5]. Among these implants, unsintered hydroxyapatite particles (uHA)/ PLLA mesh and PCL mesh has been widely used in orbital reconstruction nowadays. uHA/PLLA mesh has the benefits of bioactive and osteo-inductive properties and relative long degradation period [6]. However, due to its thermoplastic characteristics, uHA/PLLA mesh should be contoured by heating for orbital fractures [4]. Another biodegradable material, PCL, has several advantages of adequate mechanical strength, great flexibility for manipulation at room temperature, and the ability to degrade without producing harmful byproducts $[1,7]$. However, there are some concerns that PCL could cause soft tissue adhesion because of its strong biodegradability and fibrovascular ingrowth, and this mesh-type implant is not strong enough to support the orbital contents in case of orbital fractures with large defects.

From the authors' experience, the main difference between the two materials is flexibility whilst handling the mesh. In this study, we hypothesized that the different handling properties of uHA/PLLA and PCL meshes potentially affect the surgical outcomes and complications of orbital reconstruction. In order to provide better insights into the selection of biodegradable orbital implants to repair orbital wall fractures, we compared the surgical outcomes and the postoperative implantation accuracy between UHA/PLLA (Osteotrans-MX) and PCL meshes (T\&R mesh) to determine whether any differences were present, both immediately and in an early postoperative period.

\section{METHODS}

\section{Study cohorts}

A retrospective chart review was performed, and patients who underwent surgery for orbital wall fractures using PCL (T\&R mesh; Biofab Co., Ltd., Siheung, Korea) mesh and uHA/PLLA mesh (Osteotrans-MX; Teijin Medical Technologies, Osaka, Japan), between February 2017 and January 2019, at the Department of Plastic and Reconstructive Surgery in the Sanggye Paik Hospital were included in the study on informed consent. In order to increase the power of comparability, the authors excluded cases where other kinds of PLLA, PGA, and PDLLAderived orbital implants were used, as well as patients aged $<16$ years or with a history of exposure to orbital trauma or injury and ophthalmologic surgery. The surgery was determined based on the clinical evidence of orbital tissue entrapment, poor ocular motility, diplopia interfering with daily activities beyond the fifth day after the accident, estimated orbital wall defect size $>2 \mathrm{~cm}^{2}$ in the computed tomography (CT) image, or enophthalmos with a difference of $>2 \mathrm{~mm}$ using exophthalmometry [1]. All patients underwent pre- and postoperative CT using a GE LightSpeed volume CT (GE Medical System, Milwaukee, WI, USA), via continuous 3-mm-thick axial and coronal slices. We measured the curved linear line of margin of the inferomedial orbital wall in all coronal sections of the CT scan, and the results were summed and multiplied by the section thickness to obtain the total defect size. Similar to the extent of the orbital wall defect shown in a previously published our study, all included cases were classified into three categories: small, large, and extremely large, based on the preoperative CT images [1]. A small defect is defined as an isolated defect of the orbital floor or medial wall, which is less than $2 \mathrm{~cm}^{2}$. A large defect is defined as a defect of orbital floor and/or of the medial wall, which is $>2 \mathrm{~cm}^{2}$, and within the anterior two-thirds. An extremely large defect is defined as a defect of the entire orbital floor and the medial wall, extending into the posterior third. Patients were divided into the PCL and UHA/PLLA groups based on their implant type. To reduce possible bias affecting the surgical outcomes and to maintain similarity in the extent of fractures between both groups, the patients were matched for similar defect size and location, as detected in the preoperative CT images. The patients were randomly selected and individually matched into each group. And cases with combined lateral or roof orbital fractures were excluded. Finally, the study identified 30 pairs who met the inclusion criteria. This study was approved by the Institutional Review Board of the Sanggye Paik Hospital (IRB No. 2019-12-004).

\section{Treatment procedure}

The authors used conventional surgical approaches for the two implants: the sub-ciliary approach for inferior or inferomedial wall fracture and transcaruncular approach for medial wall fracture. After dissection of the soft issue and elevation of the periosteum periorbital wall, the herniated orbital contents were reduced by gently depressing the bone edges with an elevator and separating the adhesions from the sinus mucoperiosteum with complete atraumatic release and anatomic reduction of the fracture site. In the cases where it was necessary to cover the defect or protect a mobile orbital bone fragment from falling out of reduction into the sinus, an orbital implant was used. Based on individual subjective assessment of the extent and shape of the damaged orbital wall, the PCL mesh was molded 
manually and cut using scissors at room temperature, whereas the uHA/PLLA mesh was contoured after heating by dipping it into a hot water bath. Finally, the fabricated orbital implant was inserted above the fracture site under the periosteum [1].

\section{Outcome measurement statistical analysis}

The surgical outcomes of our interest were the anatomical accuracy of implant for replacement of bony defect detected in the postoperative CT image and the functional outcomes including diplopia, ocular motility, and enophthalmos. To assess the postoperative implantation accuracy, the quality of reconstruction was scored as excellent (score 2) or poor (score 1), with respect to the loss of replacement or malposition of the implant at three distinct locations using coronal postoperative CT images (directly dorsal to the orbital rim, in the middle of the reconstructed area, and slightly anterior to the end of the reconstruction).

Ocular symptoms were evaluated before and after surgery, and 6 months postoperatively. Evaluations at each visit included the assessment of ocular motility, diplopia, and enophthalmos. Other complications were divided into early and late complications and were classified as bleeding and infection/inflammatory response. We performed the Pearson chi-square test or Fisher exact test for categorical variables and the two-sample $t$-test for continuous variables. A value of $p<0.05$ was considered significant. All statistical analyses were conducted using Statistical Package for the Social Sciences IBM SPSS version 19.0 (IBM Corp., Armonk, NY, USA).

\section{RESULTS}

This study included 60 patients, with a mean age of 39.1 years, equally divided into two groups. The patient-related characteristics, such as age and sex, were similar between both groups. No differences with respect to causes of orbital fracture were observed between the two groups, and assault was the most common cause of orbital wall fracture in both groups. The orbital floor was the most common site of orbital wall fracture $(n=21$ [70\%] in the PCL group and $\mathrm{n}=17$ [57\%] in the uHA/PLLA group), followed by medial wall and inferomedial fractures $(23 \%$ and $7 \%$ in the PCL group and 33\% and 10\% in the uHA/PLLA group, respectively). Regarding the extent of orbital wall defect, small size defects were observed in 14 patients (47\%) in the PCL group and 22 patients (73\%) in the UHA/PLLA group, while the large and extremely large size defects were observed in 16 patients (53\%) in the PCL group and eight patients (27\%) in the uHA/PLLA group. There was no significant difference between the two groups in the extent of defect size $(p=0.058)$. The preop- erative evaluation showed 11 patients (36.7\%) with limited ocular motility after the injury and five patients (16.7\%) complaining of diplopia in each group. There were three patients (10\%) with enophthalmos in the PCL group but none in the uHA/ PLLA group, as described in Table $1(p=0.237)$.

Table 2 describes the functional outcomes related to the ocular symptoms during the 6-month postoperative follow-up, where none of the patients of the two groups showed any eye movement restriction. There was no diplopia or enophthalmos observed in the PCL group, but two patients with diplopia and one with enophthalmos were observed in the UHA/PLLA group. However, there was no significant difference in the ocular symptoms between both groups. During the long-term follow-up period, no infection or inflammatory changes were re-

Table 1. Demographics and fracture related data of the 2 groups

\begin{tabular}{|c|c|c|c|}
\hline Variable & $\begin{array}{c}P C L \\
(n=30)\end{array}$ & $\begin{array}{c}\text { UHA/PLLA } \\
(n=30)\end{array}$ & $p$-value \\
\hline Mean age (yr) & 40.4 & 37.8 & 0.795 \\
\hline Sex & & & 0.606 \\
\hline Male & $25(83)$ & $19(63)$ & \\
\hline Female & $5(17)$ & $11(37)$ & \\
\hline Cause of injuries & & & 0.831 \\
\hline Assault & $13(43)$ & $11(37)$ & \\
\hline Traffic accident & $6(20)$ & $6(20)$ & \\
\hline Sports accident & $4(13)$ & $7(23)$ & \\
\hline Fall & $7(23)$ & $6(20)$ & \\
\hline Location of orbital wall defect & & & 0.109 \\
\hline Medial & $7(23)$ & $10(33)$ & \\
\hline Inferomedial & $2(7)$ & $3(10)$ & \\
\hline Floor & $21(70)$ & $17(57)$ & \\
\hline Extent of orbital wall defect & & & 0.058 \\
\hline Small & $14(47)$ & $22(73)$ & \\
\hline Large & $10(33)$ & $3(10)$ & \\
\hline Extremely large & $6(20)$ & $5(17)$ & \\
\hline \multicolumn{4}{|c|}{ Related preoperative clinical features } \\
\hline Restriction of ocular motility & $11(37)$ & $11(37)$ & 1.000 \\
\hline Diplopia & $5(17)$ & $5(17)$ & 1.000 \\
\hline Enophthalmos & $3(10)$ & 0 & 0.237 \\
\hline
\end{tabular}

Values are presented as number (\%).

PCL, polycaprolactone; uHA/PLLA, unsintered hydroxyapatite/poly-L-lactic acid.

Table 2. Comparison of the functional outcomes related to the ocular symptoms at the 6-month postoperative follow-up

\begin{tabular}{lccc}
\hline Outcome & $P C L$ & uHA/PLLA & $p$-value \\
\hline Restriction of ocular motility & 0 & 0 & NA \\
Diplopia & 0 & 2 & 0.492 \\
Enophthalmos & 0 & 1 & 1.000 \\
\hline
\end{tabular}

Values are presented as number.

PCL, polycaprolactone; uHA/PLLA, unsintered hydroxyapatite/poly-L-lactic acid; NA, not applicable. 
ported in either group.

The successful anatomical replacement of the defect based on the postoperative CT images is shown in Table 3. It is revealed by the anatomical accuracy of the implant used to replace the bony defect in both groups. There were 27 patients in the PCL group and 22 patients in the uHA/PLLA group whose implants were well positioned with an appropriate orbital curvature lining. Figs. 1-4 represent excellent cases of orbital wall reconstruction with PCL implant and UHA/PLLA implant in orbital

Table 3. Comparison of the anatomical accuracy of the implant in the replacement of bony defect

\begin{tabular}{lrcc}
\hline Variable & PCL & UHA/PLLA & $p$-value \\
\hline Accuracy & & & 0.181 \\
Excellent & 27 & 22 & \\
Poor & 3 & 8 & \\
\hline
\end{tabular}

Values are presented as number. Excellent means well positioned with appropriate orbital curvature lining and poor means poorly positioned or poorly supporting soft tissue.

PCL, polycaprolactone; uHA/PLLA, unsintered hydroxyapatite/poly-L-lactic acid.
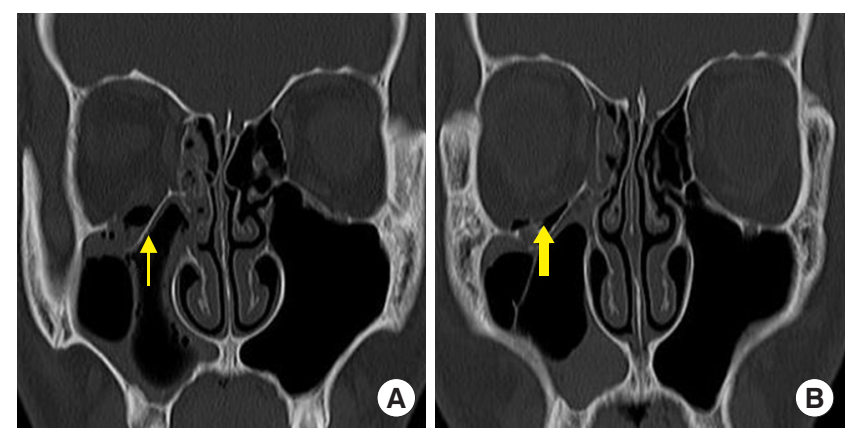

Fig. 1. An 18-year-old boy with a right orbital floor fracture owing to assault. (A) Preoperative computed tomography (CT) scan shows a right orbital floor fracture (thin arrow). (B) Postoperative CT scan shows polycaprolactone implant in the defect area of the orbital floor (thick arrow).
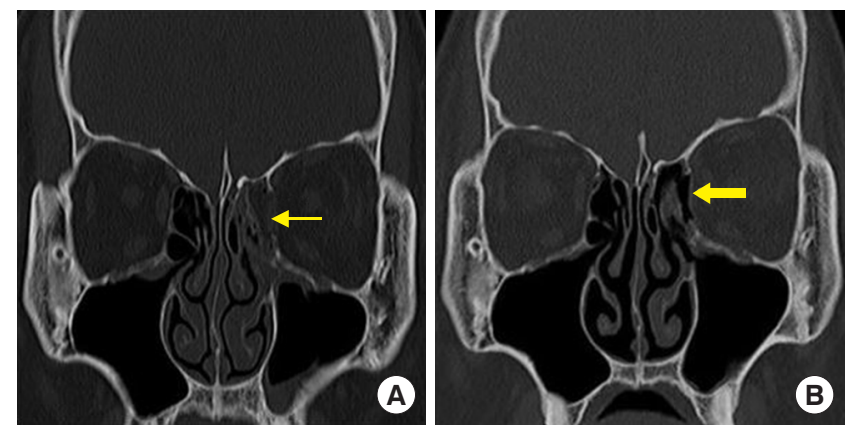

Fig. 2. A 40-year-old man with a left medial orbital wall fracture owing to assault. (A) Preoperative computed tomography (CT) scan shows a left medial wall fracture and sagging of the extraocular muscle and soft tissue (thin arrow). (B) Postoperative CT scan shows the polycaprolactone implant in the defect area of the medial orbital wall (thick arrow). floor and medial wall fractures. In the uHA/PLLA group, eight patients showed "poor" poorly positioned implants or poorly supported soft tissue, while one showed postoperative malrotation of the implant (Fig. 5). However, in the PCL group, there
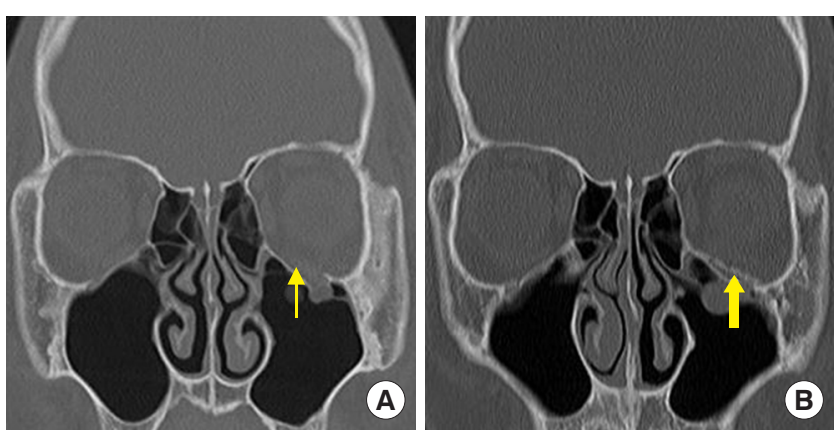

Fig. 3. A 17-year-old boy with a left orbital floor fracture owing to sports-related injury. (A) Preoperative computed tomography (CT) scan shows a left orbital floor fracture (thin arrow). (B) Postoperative CT scan shows unsintered hydroxyapatite/poly-L-lactic acid implant in the defect area of the orbital floor (thick arrow).
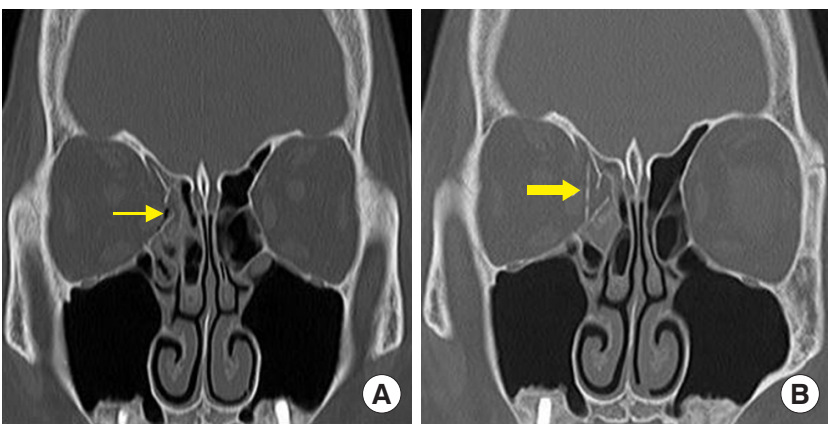

Fig. 4. A 54-year-old woman with a right medial wall fracture owing to traffic accident. (A) Preoperative computed tomography (CT) scan shows a right medial wall fracture and sagging of the extraocular muscle and soft tissue (thin arrow). (B) Postoperative CT scan shows the unsintered implant in the defect area of the medial orbital wall (thick arrow).
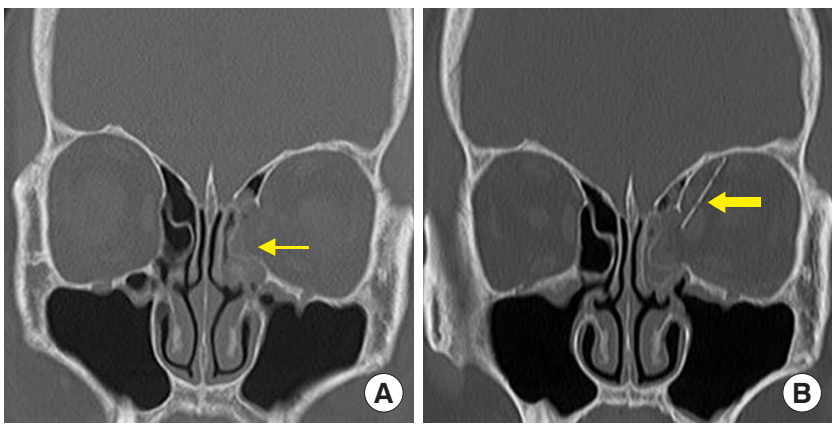

Fig. 5. A 64-year-old woman with a left medial wall owing to a fall. (A) Preoperative computed tomography (CT) scan shows a left medial wall fracture (thin arrow). (B) Postoperative CT scan shows upper malrotation of the unsintered hydroxyapatite/poly-L-lactic acid implant (thick arrow). 
were three patients with "poor" poorly positioned implants or poorly supported soft tissue, but there was no case of implant malrotation. There was no significant difference between the two groups in anatomical accuracy of the implant $(p=0.181)$.

\section{DISCUSSION}

In the reconstruction of the orbital wall fracture, the choice of the implant material usually depends on various factors, such as fracture location, defect size, surgeon preference, cost-effectiveness, and availability of each implant, because each implant has certain advantages and disadvantages. Autologous bone grafts are used because there is no concern of infection or foreign body reaction with them; however, they have the disadvantages of donor site morbidity and difficulty in handling and molding as per the orbital wall curvature [1,7]. Hence, alloplastic implants have emerged as their substitute in orbital reconstruction. Initially, non-resorbable implants such as titanium and Medpor (porous polyethylene) were used for their benefits of excellent tensile strength and support of the orbital tissue. However, these implants remain as permanent foreign objects, which means that the patients are exposed to persistent complications such as infection, inflammation, and extrusion of these implants [1,7]. As a result, the interest in biocompatible and resorbable implants has recently increased, and various materials including PDS, PLLA, PGA, PDLLA, and PCL of varying compositions have been introduced.

PDS is a semi-crystalline polymer composed of p-dioxanone and degraded by hydrolysis, which loses half of its supportive strength after 3 weeks [8]. PDS gets rapidly resorbed in 6 months, and the scar following resorption was not enough to support the globe, especially in large defects [9]. PGA is the simplest polyester and a highly crystalline material [8]; however, it degrades rapidly and its mechanical strength decreases by half in 2 weeks; thus, it needs to be copolymerized with other compounds [8]. PLLA is a semi-crystalline material with good strength and slow degradation due to its hydrophobicity [10]. As PLLA degrades slowly over 2 to 6 years by hydrolysis, it is commonly copolymerized with PGA or PDLLA in various compositions, which decides the degradation period and durability of each implant. PDLLA is a completely amorphous polymer that degrades fast, has low tensile strength, and is used as an implant in the PLLA/PDLLA compound [8]. OsteotransMX (Teijin Medical Technologies) is a bioactive and completely resorbable implant and is a compound of uHA particles (40 weight $\%$ in plates) and PLLA. HA is a mineral component of calcified tissue, and uHA particles are synthesized by hydrolysis of calcium hydrogen phosphate anhydrate in the presence of calcium carbonate [11]. Hence, uHA particles appear radiopaque in radiographs, thus permitting surgeons to identify Osteotrans orbital implants in postoperative CT images. This material provides great supportive strength comparable to that of the human cortical bone. During degradation, release of unbound uHA debris and deposition of calcium phosphates affect osteoconduction [11]. However, during the late hydrolysis and degeneration stages, by-products of PLLA like lactic acid can provoke infection and inflammation when hydrolysis debris exceed the clearing system capacity of the body. A prior study showed a case of foreign body reaction, after 2 years of surgery using uHA/PLLA plate and screw, that required plate removal in the left frontozygomatic suture, inferior orbital rim, and lateral midfacial buttress [11]. Another study showed that 13 (17\%) out of 78 patients with facial bone fracture or undergoing posterior malignant tumor reconstruction using Osteotrans experienced infection complication, and plate removal had to be performed in all of them [12].

PCL is a semi-crystalline polymer formed from $\varepsilon$-caprolactone and degraded by hydrolysis [7]. PCL mesh has several beneficial characteristics for use as implants in orbital wall reconstruction. Firstly, a PCL implant provides adequate mechanical support and degrades over 2 years, which is long enough for new bone formation in osteoconductive environments [1,7]. Secondly, PCL implants cause lesser foreign body reaction, infection, and inflammation than PLLA or PLGA implants because its byproducts after hydrolysis, like caproic acid, are weaker acid metabolites (approximate $\mathrm{pH} 5$ ) as compared to those of PLLA or PLGA implants [8]. A study showed that $98 \%$ of patients undergoing augmentation rhinoplasty using PCL mesh did not report infection or foreign body reaction until the 18-month follow-up, and regeneration of neo-cartilage tissue was observed in the histopathological analysis [13]. Lastly, due to the malleability of PCL mesh at room temperature, PCL implants can be more easily handled and transformed to suit the individual characteristics of the orbital fracture compared to uHA/PLLA implants [1,7].

Although several outcome studies on biodegradable orbital implants have been published, the majority of these have focused on the outcomes of one particular type of resorbable implant, or the comparison of polylactide- or polyglycolide-derived implants. To the best of our knowledge, there are no studies directly comparing the surgical outcomes of polylactide-derived implants and PCL biodegradable implants. In the authors' opinion, there is a significant difference between the property of uHA/PLLA and PCL meshes. This includes the materials' malleability for manipulation and molding of the mesh to fit into the orbital defect. We think that this characteristic is im- 
portant for the simulation of three-dimensional anatomical inferomedial orbital wall contours in the reconstruction of orbital wall fractures. Based on this, the authors assumed that a more accurate replacement and better surgical outcome can be expected in the PCL mesh group compared to the uHA/PLLA mesh group. This hypothesis was based on the increased malleability of PCL, which molds the implants to the internal curvature of the orbital wall and fits well into the defect, thus resulting in a better surgical outcome and less complications.

According to the results of the current study, both implants showed remarkable improvements in ocular symptoms, and there were no statistically significant differences between them with respect to surgical outcomes and postoperative complications, such as infection, inflammation, or enophthalmos. With regard to the postoperative results, the anatomical accuracy of orbital repair was measured and compared between the two groups. The difference in the percentage of patients that received an "excellent" score in anatomical accuracy was not statistically significant.

This comparative study between PCL and uHA/PLLA implant had several limitations. First, the surgical outcomes when using a biodegradable mesh, such as ocular motility, diplopia, and enophthalmos, can be affected by several factors, including material degradation time, material flexibility, hydrolysis byproducts, and even the surgeons' practical skill. Therefore, multifactorial analysis for surgical outcomes should be performed. However, due to the retrospective design of this study, patient or implant selection bias and differences in surgical skills between surgeons could not be avoided, and a multivariate analysis could not be performed due to the small sample size in each group. Secondarily, our results were derived from relatively short-term follow-ups in both groups. This accounts for any delayed onset of infection, inflammation, and ocular symptoms not being detected. Furthermore, due to this, it is unclear whether the difference between the degradation times for the two materials affected the surgical outcome in this study. Further research involving long-term, randomized, prospective studies are necessary to evaluate the associated benefits and complications in more detail. Lastly, compared to Osteotrans, PCL implants are ambiguously detected on facial CT bone scans, but PCL mesh can be detected only if the background contrast is adjusted on the CT scan. Nevertheless, to the best our knowledge, there are no studies directly comparing the surgical outcomes of polylactide-derived implants and PCL biodegradable implants.

This study demonstrates that there were neither any significant differences in the surgical outcomes like diplopia and enophthalmos nor any complications with the two well-known implants (PCL implant and uHA/PLLA implant). Although the implant choice in orbital wall reconstruction often depends on the surgeon's preference, PCL implants and uHA/PLLA implants can be used safely and have similar complications and surgical outcomes in orbital wall reconstruction.

\section{NOTES}

\section{Conflict of interest}

No potential conflict of interest relevant to this article was reported.

\section{Ethical approval}

The study was approved by the Institutional Review Board of Sanggye Paik Hospital (IRB No. 2019-12-004) and performed in accordance with the principles of the Declaration of Helsinki. Written informed consents were obtained.

\section{Patient consent}

The patients provided written informed consent for the publication and the use of their images.

\section{ORCID}

Hyeon Uk Jang https://orcid.org/0000-0003-1712-8839

So Young Kim https://orcid.org/0000-0001-5552-8003

\section{REFERENCES}

1. Kim SY. Application of the three-dimensionally printed biodegradable polycaprolactone (PCL) mesh in repair of orbital wall fractures. J Craniomaxillofac Surg 2019;47:1065-71.

2. Young SM, Sundar G, Lim TC, Lang SS, Thomas G, Amrith S. Use of bioresorbable implants for orbital fracture reconstruction. Br J Ophthalmol 2017;101:1080-5.

3. Kontio R, Suuronen R, Salonen O, Paukku P, Konttinen YT, Lindqvist C. Effectiveness of operative treatment of internal orbital wall fracture with polydioxanone implant. Int J Oral Maxillofac Surg 2001;30:278-85.

4. Chu SG, Lee JS, Lee JW, Yang JD, Chung HY, Cho BC, et al. Comparisons among four types of absorbable plates used for internal fixation of zygomaticomaxillary complex fractures. J Craniomaxillofac Surg 2019;47:383-8.

5. Choi SH, Lee JH. Absorbable plate-related infection after facial bone fracture reduction. Arch Craniofac Surg 2016;17:1-4.

6. Al-Sukhun J, Tornwall J, Lindqvist C, Kontio R. Bioresorbable poly-L/DL-lactide (P[L/DL]LA 70/30) plates are reliable for repairing large inferior orbital wall bony defects: a pilot study. J Oral Maxillofac Surg 2006;64:47-55. 
7. Teo L, Teoh SH, Liu Y, Lim L, Tan B, Schantz JT, et al. A novel bioresorbable implant for repair of orbital floor fractures. Orbit 2015;34:192-200.

8. Ramesh S, Hubschman S, Goldberg R. Resorbable implants for orbital fractures: a systematic review. Ann Plast Surg 2018;81: 372-9.

9. Baumann A, Burggasser G, Gauss N, Ewers R. Orbital floor reconstruction with an alloplastic resorbable polydioxanone sheet. Int J Oral Maxillofac Surg 2002;31:367-73.

10. Nair LS, Laurencin CT. Biodegradable polymers as biomaterials. Prog Polym Sci 2007;32:762-98.

11. Hayashi M, Muramatsu H, Sato M, Tomizuka Y, Inoue M, Yo- shimoto S. Surgical treatment of facial fracture by using unsintered hydroxyapatite particles/poly l-lactide composite device (OSTEOTRANS MX(®)): a clinical study on 17 cases. J Craniomaxillofac Surg 2013;41:783-8.

12. Tatsuta S, Hayashi M, Tokunaka R, Muramatsu H, Kadomatsu K. Analysis of the postoperative absorption process of unsintered hydroxyapatite particles/poly l-lactide composite device (OSTEOTRANS MX(®)) for facial bone fractures in 13 cases. Plast Reconstr Surg Glob Open 2018;6:10-1.

13. Park YJ, Cha JH, Bang SI, Kim SY. Clinical application of threedimensionally printed biomaterial polycaprolactone (PCL) in augmentation rhinoplasty. Aesthetic Plast Surg 2019;43:437-46. 